\title{
Phytol-derived novel isoprenoid immunostimulants
}

\section{Roshni Roy Chowdhury and Swapan K. Ghosh*}

Department of Biology, Indiana State University, Terre Haute, IN, USA

\section{Edited by:}

José Mordoh, Fundación Instituto

Leloir, Argentina

Reviewed by:

Yasmin Thanavala, Roswell Park

Cancer Institute, USA

María Marcela Barrio, Fundación

Cáncer, Argentina

*Correspondence:

Swapan K. Ghosh, Department of

Biology, Indiana State University, Terre

Haute, IN 47809, USA

e-mail:sghosh@indstate.edu
This review describes the adjuvanticity of novel diterpenoids (synthetic phytol derivatives) compared to some commercially available adjuvants. The efficacy of the phytol-derived immunostimulants was evaluated in terms of their ability to activate innate immunity, amplify various antigen-specific immune responses, and engender immunological memory with no discernible adverse effects in both competent and immune-deficient mice. The profile that emerges out of these studies reveals that the phytol derivatives are excellent immunostimulants, superior to a number of commercial adjuvants in terms of long-term memory induction and activation of both innate and acquired immunity. Additionally, the phytol-derived compounds have no cumulative inflammatory or toxic effects even in immuno-compromised mice.

Keywords: immunostimulants, phytol derivatives, isoprenoid adjuvants, activation of innate immunity, vaccine efficacy, cytokine microenvironment, inflammasome, antibody and CTL response
"The art of healing comes from nature and not from the physician.

Therefore, the physician must start from nature with an open mind." -Paracelsus

Immunostimulants and immunomodulators abound in nature. All through human history, vast arrays of natural compounds, particularly those from plant and microbial sources, have provided a wealth of immunomodulators. In many ways, they help preserve all life forms and their relations, even in the midst of adversities and mutual antagonisms. Despite being strangers or dangerous, both commensals and harmful agents can unleash modulators to enrich their hosts' immune repertoire and bolster the immune system (McKee et al., 2010; Norton et al., 2010). Modulators or immunoadjuvants from simple inorganic salts like alum (Marrack et al., 2009) to bacterial cell wall constituents (Lederer, 1980; Strominger, 2007) have all been found to enhance vaccine efficacy. Chemical modifications and syntheses become necessary to improve immunoadjuvants or modulators and to unravel structure-function relationship (Azuma, 1992; Spanedda et al., 2010). The fact that the immunogenicity of a substance depends largely on the use of adjuvants was observed almost a century ago. However, the underlying mechanism still defies clarity, primarily because of the empirical and diverse nature that surrounds the selection and assessment of immunoadjuvants. No wonder, adjuvants have come to be regarded as "immunologists' dirty tricks" (Janeway, 1989).

A group of compounds that has in recent years garnered lots of interest in the field of adjuvants, are terpenoids, specifically lipophilic squalene, a triterpene (Benísek et al., 2004), and QS21, a hydrophilic triterpene glycoside (Soltysik et al., 1995; Kensil and Kammer, 1998). Terpenoids or terpenes comprise the largest and most diverse class of secondary metabolites (such as squalene involved in cholesterol biosynthesis); approximately 55,000 compounds have been identified to date (Maimone and Baran, 2007). While the enzymes responsible for terpene synthesis are found in all classes of microorganisms, plants, and animals, terpenes and terpene-derived structures are most widely synthesized by higher plants (Zwenger and Basu, 2008). All classes of terpenes are synthesized from $\mathrm{C} 5$ isoprene units that have been linked end-to-end. Monoterpenes (C10) and sesquiterpenes (C15) are often flavor and fragrance components of essential oils. In contrast, the carotenoids (C40) are yellow to red plant pigments, while the photosynthetic green pigment chlorophyll is composed of a magnesium-containing chlorin ring and a long side-chain containing the acyclic diterpenoid, phytol (C20). Plant essential oils, which are complex mixtures of volatile monoterpenes and sesquiterpenes, protect plants from disease, infestation, and predation. These oils have also been shown to have antimicrobial, anti-parasitic, insecticidal, and antioxidant/pro-oxidant activities (Isman, 2006; Bakkali et al., 2008). Many plant terpenoids are cytotoxic to tumor cells, making them useful chemotherapeutic or chemopreventive compounds (Mo and Elson, 2004; Thoppil and Bishayee, 2011). Paclitaxel (Taxol) and related taxanes are the most well known anti-cancer agents. The anti-cancer activities of some terpenoids are based on their ability to undergo protein prenylation (Bifulco, 2005). Some plant terpenoids have activities that make them useful research tools, for example in developing new drug delivery systems (Shieh et al., 1995; Kazanietz, 2005).

Based on their biological activities, terpenoids can be broadly classified into three categories: cellular function, defense, and communication. For example, terpenoids are involved in biosynthetic pathways such as cholesterol, in defense as in toxins or repellents, and in communications as hormones in aggressions and alarm pheromones (Pickett and Gibson, 1983; Harrewijn et al., 2001). Many natural isoprenoids like squalene and vitamin $\mathrm{E}$ are known for their beneficial effects on the immune system. A number of excellent reviews on squalene-based MF59 adjuvant have appeared in recent years (Reddy and Couvreur, 2009; Kalvodova, 2010; Seubert et al., 2011). Squalene is also a constituent of commercial adjuvants like Titermax, and Ribi's adjuvant. From this prospective, it is therefore quite understandable that many of these 
compounds from plant and animals have found wide usage in the rational design of pharmaceuticals and immunological adjuvants. However it is inconceivable that a single compound could fit all that is expected of a versatile immunomodulator/adjuvant. It is critical to develop well-defined, non-toxic adjuvants that would function as cocktails and augment the immunogenicity of diverse immunogens.

Epidemiological studies have suggested the importance of green vegetables in diets in order to improve resistance to infection and thus enhance immunity (Elson and Yu, 1994). Chlorophylls, found in all green vegetables, constitute an important source of an isoprenoid component, phytol (3, 7, 11, 15-tetramethyl2-hexadecen-1-ol, $\left.\mathrm{C}_{20} \mathrm{H}_{40} \mathrm{O}\right)$. It is an acyclic monounsaturated diterpene alcohol, present in vitamin $\mathrm{K}$, vitamin $\mathrm{E}$, and other tocopherols. Because phytol and other isoprenoids are lipophilic, they are capable of interacting with cell membranes as well as other lipophilic substances and metabolites (Glomset et al., 1990; Rilling et al., 1990). This ability makes these compounds suitable for use as immune adjuvants, the outcomes of which may lie in efficient delivery of antigens to antigen-presenting cells, up-regulation of costimulatory molecules and promotion of cellular crosstalk. We have been specifically interested in phytol since it is structurally simple, easily available, and cost-effective. Additionally, it was reasoned that because of regular dietary intake, phytol should be well tolerated by the body. These considerations led us to assess the adjuvanticity of phytol and its synthetic derivatives in mice and report their efficacy in vaccine formulations (Lim et al., 2006a,b; Aachoui et al., 2011a; Ghosh, 2012).

\section{WHY USE PHYTOL DERIVATIVES AS ADJUVANTS?}

Medium-length straight chain hydrocarbons (optimum 12 carbons) have previously been shown to be the most potent adjuvants (Reeves et al., 2009). Mineral or hydrocarbon oils cause inflammation and augment immune responsiveness. This finding led to the use of mineral oil-in-water emulsions as immunoadjuvants for several decades (Wilner et al., 1963). Complete Freund's adjuvant (CFA) that contains mycobacterial protein in addition to mineral oil is one of the most potent adjuvants known. However, because of its unfavorable risk/benefit ratio, use of CFA in humans has been prohibited (Satoh et al., 2003; Kuroda et al., 2004). On the contrary, oil-in-water adjuvant, which was licensed for human use in Europe almost a decade ago, is an isoprenoid, squalene-based MF59. Although MF59 is safe, some of its components including squalene were found to be arthritogenic in rodents (Holm et al., 2004). This issue has raised questions about the safety of MF59 and may restrict its use in vaccines in the future. Pristane $(2,6,10,14-$ tetramethylpentadecane) is another naturally occurring saturated hydrocarbon oil, which has been tested for its adjuvanticity. It is an isoprenoid alkane that is derived primarily from the metabolism of phytol. However, like CFA, pristane can cause a number of adverse side effects including development of plasmacytomas and generation of autoimmune conditions such as lupus and arthritis (Holmdahl et al., 2001). Currently, many other adjuvants have been described with variable safety and immunostimulation records. However, despite these efforts, aluminum salt/gel-based (alum) adjuvants remain the only standard versatile adjuvant licensed for human use in the United States. Even alum has a number of drawbacks. Alum cannot induce a $\mathrm{T}$ helper type 1 (Th1) cell-mediated immune response that is important in fighting certain viruses, bacteria, and parasites (Brewer et al., 1999). It has also been implicated in dementia, a loss of brain function that occurs with certain diseases (Marchkesbery et al., 1981). Therefore, the goal of our laboratory has been to develop safer and more broadly applicable adjuvants from natural compounds like terpenoids that meet all the hallmarks of an ideal adjuvant outlined in Figure 1.

Our studies revealed phytol to be a potent adjuvant but not without side effects. Adverse effects induced by phytol include splenomegaly, hepatotoxicity, and tumor promotion in rodents (Kagoura et al., 1999). Prior studies have also linked phytol to Refsum disease, an autosomal recessive disorder that results from the accumulation of unmetabolizable phytanic acid in tissues (Baxter, 1968). In order to overcome known and hitherto unknown shortcomings of phytol, we developed novel adjuvants by introducing chemical modifications to phytol; the goal was to improve their safety and enhance their adjuvanticity. Phytanol, which we named PHIS-01 (phytol-based immunostimulants 01 ), was obtained by hydrogenation of phytol. This modification not only reduced toxicity but also enhanced adjuvanticity. Indeed, PHIS-01 proved more potent and less toxic than phytol. This observation led us to question: (1) the importance of the phytyl moiety and (2) the nature of functional groups in adjuvanticity of synthetic phytol derivatives. These issues paved the way to the generation of a number of novel phytol derivatives (as shown in Figure 2), where the terminal -OH is modified by amination, producing phytanyl amine (PHIS02), mannosylation producing phytanyl mannose (PHIS-03) and halogenations producing phytanyl bromide, phytanyl chloride, phytanyl fluoride, and phytanyl iodide. All of these derivatives have three chiral centers and are racemic at all three. Further modifications for better adjuvanticity are currently in progress. Our objective has been to understand how the various side groups affect the adjuvanticity, the dosage required for adjuvanticity visà-vis toxicity, effects on microenvironments surrounding innate and acquired immunity and the amplitude of specific immune responses. The importance of the phytyl moiety in adjuvanticity was demonstrated by assessing the booster effects of phytane relative to phytol derivatives and some commercial adjuvants (Ghosh, 2012).

\section{RATIONALE BEHIND SPECIFIC CHEMICAL MODIFICATIONS}

Chemical modification of adjuvants to improve their effectiveness is not new, but only limited reports have appeared. The objective of these modifications has generally been to produce effective and safer products and to understand structural intricacies required for adjuvanticity. For example, bacterial lipopolysaccharide (LPS) is toxic, but it has excellent ability to mobilize innate immunity by virtue of Toll-like and other receptors, and promote maturation of dendritic cells (Yoshino et al., 2000). However, its modified version monophosphoryl lipid A (MPL) is less toxic but a better adjuvant (Salkowski et al., 1997; Thompson et al., 2005). In Quillaja saponin adjuvant, introduction of an aldehyde group promotes a Th1 type cellular response against virus or cancer (Rhodes et al., 1995; Marciani et al., 2000; Thompson et al., 2005) whereas the deacylated saponin, lacking the aldehyde functional 


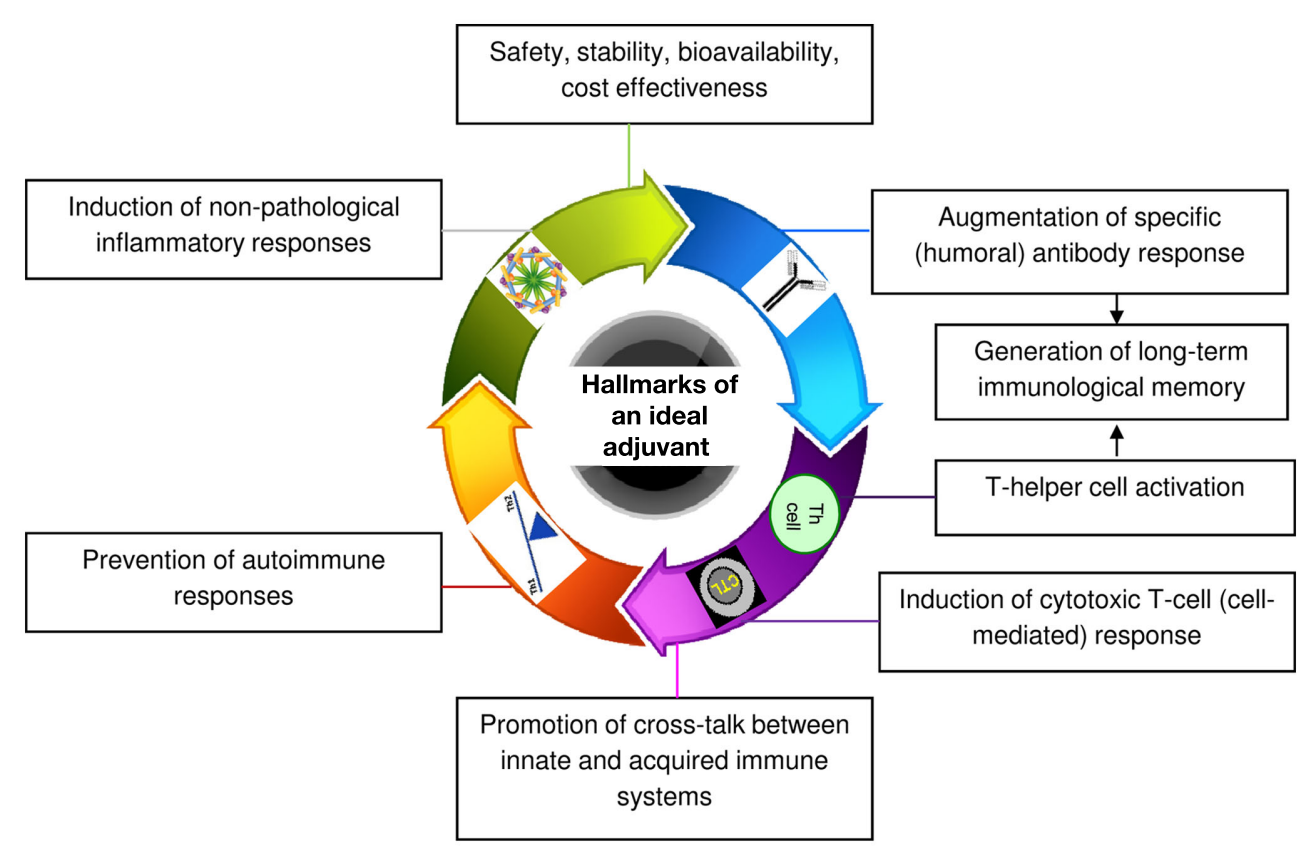

FIGURE 1 | Schematic diagram showing eight hallmark features of an ideal adjuvant.

group, favors a Th2-mediated antibody response (Marciani et al., 2001).

We hypothesized that the polar terminal moiety has a profound effect on the physico-chemical properties and therefore on the adjuvanticity of a compound. The physico-chemical properties necessary for immunomodulation by oil-in-water adjuvants are not fully understood. These emulsions function in many capacities from membrane anchoring to cell signaling. PHIS-01 has a -OH terminal group that is negatively charged. Halogenations of the terminal group increase its electronegativity, making the molecule more hydrophilic. Hydrophilicity possibly plays a role in the uptake and retention of the antigen by immune cells. Mannosylation further increases the electronegativity due to the presence of multiple $\mathrm{OH}$ groups in PHIS-03. Amination of phytol was performed to analyze the effects of a positively charged terminal residue $\left(-\mathrm{NH}_{2}\right)$ on the adjuvanticity of compounds. Differences in the modes of actions of these various adjuvants in terms of $\mathrm{T}$ cell bias (modification of the polar terminus of PHIS-01 with a hydrophilic mannose moiety (PHIS-03) favors $\mathrm{T}$ helper type 2 rather than the $\mathrm{T}$ helper type 1 response induced by PHIS01; Aachoui et al., 2011b). These findings support the fact that the nature of the polar end group in these compounds is an important modifier of adjuvanticity. An exchange of the simple alcohol or amine moiety with a mannosyl group can markedly alter the type of immune response elicited. Additional mannosylations may have entirely different effects on the phytyl moiety in terms of solubility and adjuvanticity; this aspect is currently being addressed. Another question addressed in our studies was how the polar terminus affects adjuvanticity in terms of host microenvironment (cytokine/chemokine milieu) and safety profile. Among the phytol derivatives, PHIS-01 and the halogenated phytanyl compounds were found to have no toxicity even at high doses. PHIS-01 is highly effective over a wider range of concentrations (4-44 mg/mouse). PHIS-02 functions at a much lower concentration $(2.5 \mathrm{mg} / \mathrm{mouse})$, and PHIS-03 works effectively at an intermediate dose ( $5 \mathrm{mg} /$ mouse). However both PHIS02 and PHIS-03 showed toxicity at higher doses $\left(\mathrm{LD}_{50}\right.$ values for these compounds are 5 and $10 \mathrm{mg} /$ mouse respectively; Aachoui et al., 2011a). The fact that some phytol derivatives are effective adjuvants at relatively lower doses should be good news since host exposure to the chemicals is minimal. The study of adjuvanticity in mouse models is convenient and important, because it permits the evaluation of not only histopathological toxicity and adjuvanticity but also tumorigenicity. In this context, the phytol derivatives mentioned above, have no tumorigenic properties.

\section{COMPARATIVE EFFECTIVENESS OF PHYTOL ISOPRENOIDS AND SOME COMMERCIAL ADJUVANTS}

From Table 1, it is clear that numerous challenges exist in the development of safe and versatile immunostimulants. Some of these challenges include the ability to minimize inflammation and induce both robust immune response and long-term memory. It is highly unlikely that a single immunoadjuvant will fulfill all that is needed for a non-toxic, broad and long lasting immunity. Compounds that have been proven safe, like alum, can be effective in eliciting only one arm of the immune response, limiting their use as versatile adjuvants. The art of making vaccines will undoubtedly be simpler with the understanding of the structural basis of adjuvanticity, which will eliminate the shroud of empiricism that currently exists in their selection. Moreover, this will pave the way to the generation of cocktail adjuvants for broader applications. An 


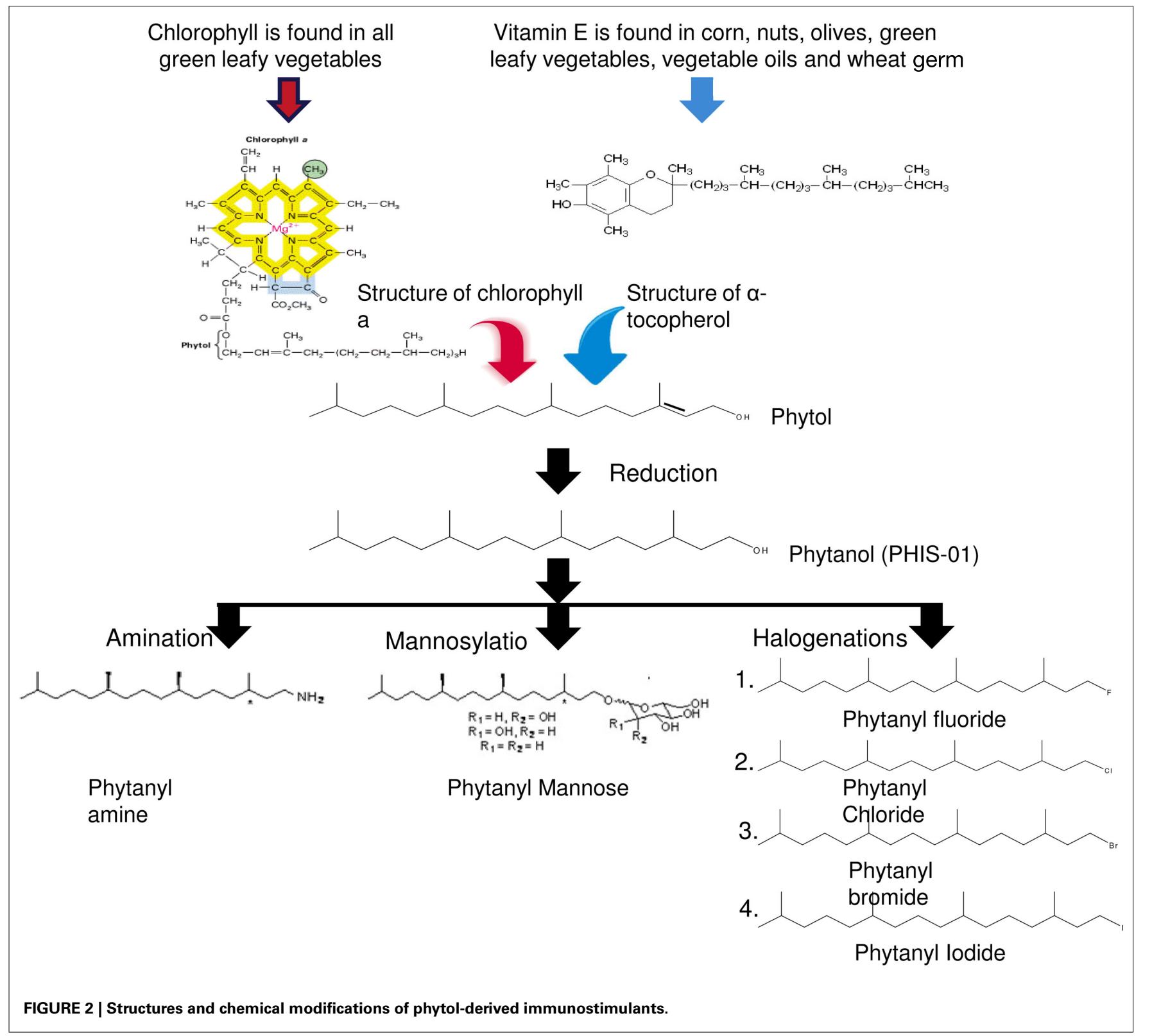

overview of the modes of actions of some commercially available immunostimulants and the phytol derivatives is given below in Table 2.

\section{MODES OF ACTION OF PHYTOL-DERIVED ADJUVANTS}

The adjuvanticity of compounds as listed in Table 2, is shaped not only by their molecular nature but also by the nature of the associated antigen (vaccine; Staats and Ennis, 1999), routes of administration (Petrovsky and Aguilar, 2004), and the vertebrate hosts (Casadevall and Pirofski, 1998). The efficiency of an adjuvant can be ascertained by its potential to boost immune responses against a variety of antigens. This is because the offending agents are not always pathogens. The threats may come from toxins, cancers, pollens, and many soluble and insoluble particulates. The list and nature of offending agents is continuously expanding and even includes addictive compounds such as cocaine. Even though vaccines have greatly impacted global health, fears persist about their potential role in the development of autoimmune and various adverse symptoms. Hence, vaccine components like immunogens and adjuvants require critical evaluation not only in healthy subjects, but also in those who are genetically averse to vaccine constituents, or in other words, populations representing varying degrees of immune competence.

Similarly, the efficacy of adjuvants can be markedly different depending on the routes of administration. For example, alum is usually more effective when administered through intradermal or subcutaneous routes than through intramuscular routes. However, due to local toxicity, alum is generally only used intramuscularly (Butler et al., 1969; Straw et al., 1985). 
Table 1 | Adjuvanticity of phytol and its derivatives compared to commercially available adjuvants.

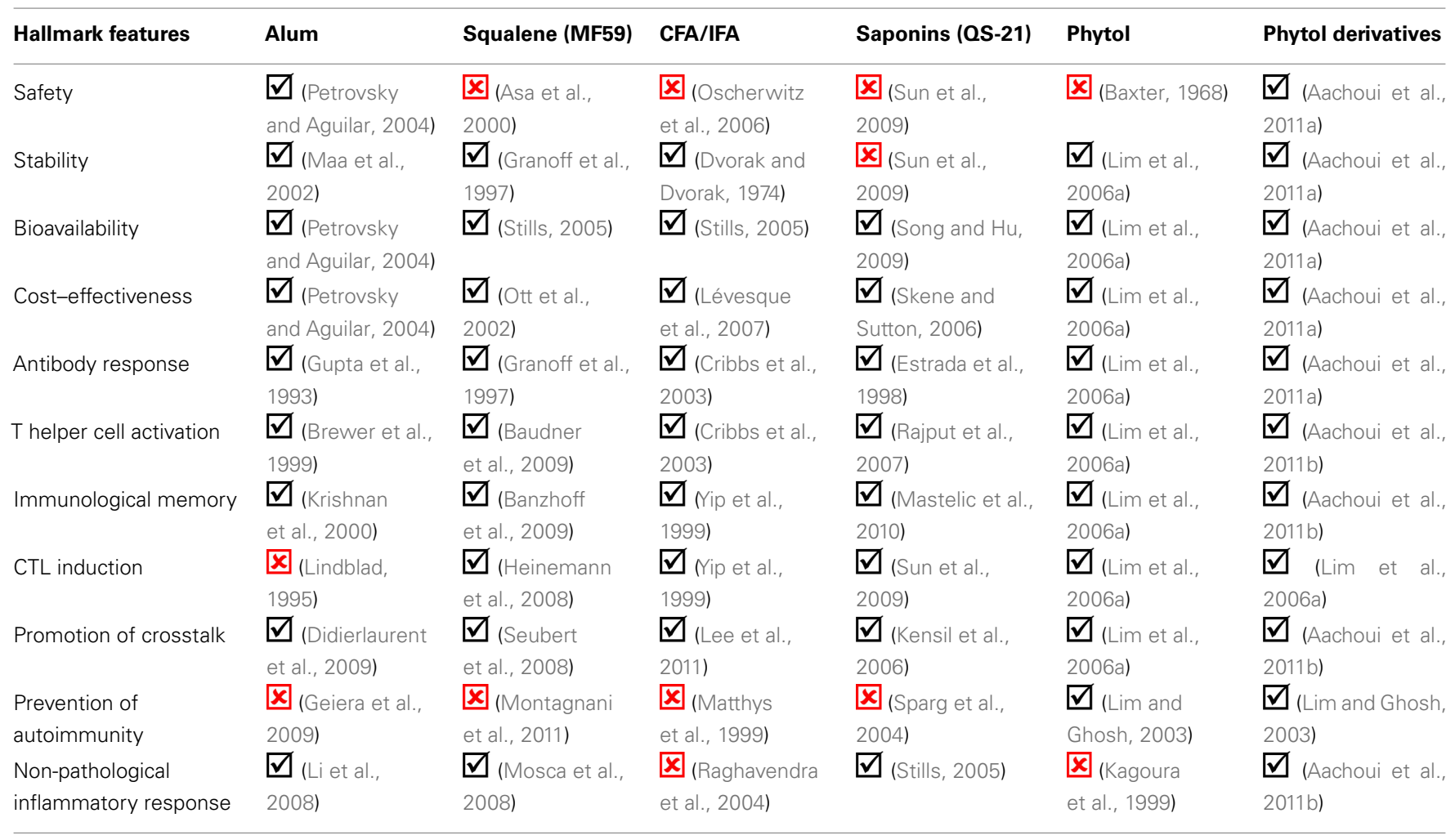

Legend: $\mathbf{x}$ Inadequate feature; $\mathbb{\square}$ Effective feature

The adjuvanticity of the phytol-derived compounds was assessed in the context of the following biological effects:

i. Minimal effective doses to overcome cumulative toxic effects, if any

ii. Amplitude and quality (isotype) of specific antibody response

iii. Crosstalk between innate and acquired immunity and modulation of cytokine chemokine microenvironments

iv. Nature of inflammatory responses

v. Generation of long-term immunological memory

vi. Prevention of autoimmune responses

\section{EVALUATION OF IN VIVO TOXICITY}

As mentioned earlier, the phytol derivatives are non-toxic and have no cumulative debilitating effects like development of plasmacytomas or arthritis (Aachoui et al., 2011a), as observed with some commercial adjuvants (Kagoura et al., 1999; Matthys et al., 1999).

\section{INDUCTION OF SPECIFIC HUMORAL IMMUNE RESPONSE}

Keeping in mind the knowledge about the empiricism in the choice of adjuvants, we tested the phytol-derived adjuvants in mice (BALB/c and C57BL/6) against a variety of antigens including: (1) a standard protein antigen (Ovalbumin), (2) a hapten-protein conjugate (Phthalate-Keyhole Limpet Hemocyanin), (3) a Grampositive bacterial pathogen (Staphylococcus aureus), (4) a Gramnegative bacterial pathogen (Escherichia coli), and (5) Tumor antigens (2C3, A20 B-lymphomas and EG7-OVA, T-lymphoma). We also tested efficacy of some phytol adjuvants admixed with protein antigens in New Zealand white rabbits. The efficacy of the phytol derivatives was compared to a number of different commercially available adjuvants like alum, complete, and in CFAs and squalene in different sets of experiments. Our findings reveal that the phytol derivatives consistently evoke high titer antibody response against the various antigens, promote efficient class switching and are better than the commercial adjuvants tested (Lim et al., 2006a,b; Li, 2009; Aachoui, 2011).

The robustness of the antibody response induced in the various adjuvant-treated groups was evaluated in terms of isotype switching, since it is as a way to ascertain the involvement of T helper cells. Our results showed that the phytol-derived adjuvants induced all IgG sub classes, in much the same way as IFA, the most potent commercial adjuvant known. The control group with no adjuvant yielded only IgG1 type antibody, while all adjuvant-treated groups induced IgG2a, IgG2b, and IgG3 in addition to IgG1. Elicitation of these various IgG subclasses testified the promotion of $\mathrm{T}$ helper cell polarization by the test adjuvants (Aachoui et al., 2011a,b). The phytol derivatives were also found to induce long-term immunological memory irrespective of the nature of the antigen (Lim et al., 2006b).

In order to investigate the efficacy of the phytol compounds in inducing antigen-specific cytotoxic T-lymphocytes (CTL), two different tumor models, namely E.G7-OVA and 2C3 were used in $\mathrm{C} 57 \mathrm{BL} / 6$ and $\mathrm{BALB} / \mathrm{c}$ mice respectively. The results showed that phytol derivatives in vaccine formulations, unlike 
Table 2 | Comparison of the modes of action of phytol derivatives with other common adjuvants.

\begin{tabular}{|c|c|c|}
\hline Immunostimulants & Modes of actions & References \\
\hline \multirow[t]{6}{*}{ Alum } & Induction Th2-type immune response characterized by lgG1 isotype antibodies & Lindblad (1995) \\
\hline & Danger model*: induction of necrosis and release of uric acid & Allan (2008) \\
\hline & Activation of Nlrp3 inflammasome leading to the release of pro-inflammatory cytokines & Li et al. (2008) \\
\hline & Clonal expansion of antigen-specific T cells and B cell priming & Smith et al. (2004) \\
\hline & Formation of antigen depot leading to its slow release & Blagowechensky (1938) \\
\hline & Direct interaction with dendritic cell surface lipids & Mbow et al. (2011) \\
\hline \multirow[t]{3}{*}{ MF59 } & Induction of both Th1 and Th2-type immune responses & Traquina et al. (1996) \\
\hline & Recruitment and activation of APCs & Banzhoff et al. (2008) \\
\hline & Recruitment of innate immune cells like monocytes and granulocytes & Seubert et al. (2008) \\
\hline \multirow[t]{4}{*}{ Freund's adjuvants } & Induction of both Th1 (CFA ${ }^{\#}$ ) and Th2-type immune responses (IFA ${ }^{\S}$ ) & Billiau and Matthys (2001) \\
\hline & Stimulation of localized inflammations & Matthys et al. (1999) \\
\hline & Mycobacterium in CFA act as PAMPs ${ }^{+}$and can activate Toll-like receptors & $\operatorname{Lim}(2003)$ \\
\hline & Recruitment and activation of APCs & Mclnerney et al. (1991) \\
\hline \multirow[t]{2}{*}{ Saponins } & Induction of CTL response (both Th1 and Th2 cytokines) & Oda et al. (2000) \\
\hline & Promotion of antigen presentation to APCs? & Cox et al. (1998) \\
\hline \multirow[t]{3}{*}{ Phytol derivatives } & Induction of both Th1 and Th2 responses & Aachoui et al. (2011b) \\
\hline & Induction of CTL response & Lim et al. (2006a) \\
\hline & Activation of the NIrp3 inflammasome leading to the release of pro-inflammatory cytokines & Aachoui et al. (2011b) \\
\hline
\end{tabular}

*Danger signal hypothesis (Kono and Rock, 2008).

+ PAMPS, pathogen associated molecular patterns (Kono and Rock, 2008).

"NIrp3, Nod-like receptor protein 3.

${ }^{*}$ CFA, Complete Freund's adjuvant.

§IFA, Incomplete Freund's adjuvant.

?APCs, Antigen-presenting cells.

Alum, could elicit tumor-specific cell-mediated effector activity. Phytol and PHIS-01 were also found to significantly augment idiotype-specific CTL responses (Lim et al., 2006a).

These results clearly established that the phytol derivatives were not only potent elicitors of the humoral immune response, but also were effective against diverse groups of antigens. In this regard, the phytol derivatives are better immunostimulants than alum, which is ineffective against several antigens and fail to induce antitumor CTL responses.

The efficacy of phytol derivatives against viral antigens has not been tested yet, but this definitely remains an area of future interest. Since some of the phytol-derived compounds have shown significant CTL induction, we believe that they could be useful in the development of viral vaccines.

\section{MOBILIZATION OF BIO-RESPONSE MODIFIERS AND INDUCTION OF INFLAMMATORY RESPONSES}

The importance of chemical bonds and functional moieties in shaping the adjuvanticity of terpenoids, like phytol derivatives, can be discerned from the ability of these compounds to polarize the immune system by mobilizing myriad bio-response modifiers. Cytokines and chemokines are bio-response modifiers, because they determine the immune microenvironment by orchestrating the recruitment and interaction of cells belonging to both innate and acquired immunity. Cytokine/chemokine arrays performed for PHIS-01, PHIS-02, and PHIS-03 revealed that PHIS-01 and PHIS-03 triggered a polarization of T cell response that developed
$24 \mathrm{~h}$ post-injection (Aachoui et al., 2011b). PHIS-01 induced a Th1 response while PHIS-03 promoted a Th2 response (Aachoui et al., 2011b). PHIS-02 had a profile similar to that of PHIS-01. This type of T-lymphocyte polarization can have great implications in the development of successful cocktail immunostimulants. Among the phytol derivatives tested, PHIS-01 was the most potent in evoking T cell activating chemokines like RANTES, TCA-3, and cytokines like IL-12 and IL-4.

Expression of chemokines like KC (CXCL1, murine equivalent of IL-8), LIX (CXCL5), and MCP-1 (CCL2) was high in all adjuvanted groups including alum, although the effect was much more pronounced with the phytol-based immunostimulants. Surges in the expression of these chemokines reflect the mobilization of neutrophils, eosinophils, and monocytes. The phytol derivatives, unlike alum, also induced the secretion of the growth factor G-CSF. In contrast to all other groups, PHIS-01 showed enhanced expression of chemokines like SDF-1, MIG, Fas ligand, and Fractalkine. These results proved PHIS-01 to be the most effective in eliciting a full-bodied immune response by activating cells of both the innate and acquired immunity.

Both the adjuvant and the antigen shape the immune microenvironment. In order to understand how the dynamics of the cytokine microenvironment change in the presence of an antigen, we assessed the cytokine milieu at peritoneal sites $24 \mathrm{~h}$ after administration of soluble protein antigens KLH and OVA with or without alum or PHIS-01, PHIS-02, and PHIS-03. Our results showed that when the adjuvants were mixed with the antigens KLH and OVA, a 
large pool of cytokines and chemokines (notably, BLC, Eotaxin-2, LIX, MCP-1, MIP-1 $\gamma$, TCA-3, M-CSF, IL-4, IL-12p40p70, IL-1 $\alpha$, sTNFRI, sTNFRII) was induced, thereby further impacting the cytokine/chemokine microenvironment (Aachoui et al., 2011b). However, what percentage of the eventual cytokine/chemokine microenvironment, is contributed by the antigen or the adjuvant in the mix, remains unclear. Both antigens and adjuvants have their respective molecular signatures, which may largely be different from the molecular signatures when the antigens and adjuvants are emulsified together. The host immune environment is the net outcome from the combined contributions of both adjuvants and immunogens.

The cytokine milieu plays a significant role in setting the pace and overcoming the barriers of $\mathrm{CD} 4{ }^{+} \mathrm{CD} 25^{+}$regulatory $\mathrm{T}$ cells (T-regs; Medzhitov and Pasare, 2003; Pasare and Medzhitov, 2004). The efficacy of standard and experimental adjuvants therefore needs to be assessed in terms of their ability to lower the impediments posed by T-regs and other immunoregulatory mechanisms. This is critical, particularly in order to provoke antitumor immunity. It is not clear whether overcoming barriers due to immune tolerance to tumor requires kinds of adjuvants that induce inflammatory response. Our experience with phytol derivatives, particularly PHIS-01, suggests that inflammation is not a prerequisite in overcoming immune refractoriness of the host.

Our experiments revealed that the phytol derivatives function by exerting apoptotic/necrotic effects on target cells. However, these apoptotic/necrotic effects induced by the phytol derivatives did not persist as happens with Freund's adjuvants (Aachoui et al., 2011a). We therefore hypothesized that the efficiency of the phytol-based adjuvants depended on their ability to induce non-pathological inflammatory reactions, elaborating cytokines and chemokines capable of the recruitment and activation of APCs. In order to test this hypothesis, we analyzed the expression patterns of 84 genes associated with various inflammasome pathways. Our results showed that like alum, the phytol-based immunostimulants boost the expression of the NLRP gene family, specifically NLRP3 and other inflammasome complexes (Aachoui et al., 2011b). PHIS-01 in particular, activated the genes of the NLRP3 Inflammasome, suggesting a mode of action similar to that of alum even though the latter does not induce CTL response (Lindblad, 1995).

Our cytokine/chemokine and inflammasome array data together indicate that the phytol compounds initiate and activate both the innate and acquired immunity by cell recruitment, increased endocytosis in monocytes, stimulation of monocyte differentiation into macrophage or DC, and activation of $\mathrm{T}$ cells. PHIS-01 is more potent than PHIS-03 in the induction of multiple Nod-like receptors, cytokines/chemokines and downstream signaling molecules. This finding may account for the ability of PHIS-01 to activate both the Th1 and Th2 responses in contrast to PHIS-03, which only activates a Th2 response. From our studies, it is apparent that innate immunity plays an important role in the adjuvanticity of phytol derivatives. Both PHIS-01 and PHIS03 activated a cluster of molecules such as IL-1 $\alpha$, Timp-1, Cflar, Bir3, and XIAP, that function as tissue injury response molecules or for blocking of apoptosis. PHIS-01 was a very potent inducer of these genes, which implied a higher apoptotic/necrotic activity; as a result of which, there could be significant release of danger signal molecules from the surrounding tissues leading to the activation of innate immunity. Based on the information gathered from our cytokine/chemokine and inflammasome arrays, we predicted a putative signaling pathway (Figure 3) that could delineate the mode of action of phytol-based immunostimulants.

The study of adjuvants and their efficacy or versatility leaves us with the following questions: (1) Can adjuvants overcome immunological anergy by triggering inflammasomes (that eventually lead to activation of NLRP or IL-1)? (2) Do specific immunity and its robustness depend on non-pathological inflammation initiated by adjuvants?

\section{PREVENTION OF AUTOIMMUNE RESPONSES}

The success of an immunostimulant depends not only on its ability to elicit a robust immune response, but also its safety and wide applicability. A major concern that persists about immunostimulants is their potential role in autoimmune reactions. Evaluation of immunostimulants in autoimmune-prone animal models is therefore important in vaccine development. Our previous studies have shown that phthalate, as a conjugate or as DEHP (diethyl hexyl phthalate, a plasticizer), can induce cross reactive antiDNA antibody response and promote lupus-like syndromes in NZB/WF1 mice (Lim and Ghosh, 2003, 2004). This ability of phthalate to induce both anti-phthalate and cross reactive antiDNA antibodies was, therefore, utilized to assess the efficacy and safety of the phytol derivatives (PHIS-01, PHIS-03) relative to alum. We studied phthalate-specific antibody response, antiDNA response, and other parameters of autoimmune disorder. We also assessed antibody isotype and cytokine/chemokine profile induced.

Pristane, an isoprenoid adjuvant like the phytol derivatives, has been shown to promote lupus-like syndromes and pathological nephritis in both autoimmune-prone and non-susceptible mouse strains after a single intra-peritoneal injection. However, in spite of their structural similarities to pristane, the phytol derivatives did not induce any adverse autoimmune symptoms. Additionally, squalene, a triterpene (similar to the diterpene phytol derivatives), and Freunds' adjuvants (CFA/IFA) are also known to provoke lupus-like syndromes in non-autoimmune-prone BALB/c mice (Satoh et al., 2003).

An imbalance between the Th1 and Th 2 cytokines is considered a hallmark of lupus. In a previous study, it has been shown that hydrocarbon oil adjuvants like pristane can induce an overproduction of Th1 polarizing cytokines like INF- $\gamma$ (Peng et al., 1997). This and high levels of IL- 6 and TNF- $\alpha$ can aggravate lupus-like diseases in rodents. NZB/WF1 mice immunized with phthalate$\mathrm{KLH}$ alone or with adjuvants induced moderate expressions of both Th1 (IFN- $\gamma$, IL-12) and Th2 (IL-3, -4, -10, and -13) cytokines, and low but detectable levels of pro-inflammatory cytokines (IL-1, IL-6, IL-17, TNF- $\alpha$ ). All adjuvant-treated groups registered high levels of anti-inflammatory cytokines TNFRII, TNFRI, TIMP-1. Interestingly, the Th1 and Th2 responses generated by different adjuvants was directed more toward phthalate as evident by induction of the IgG subclass, whereas the cross reactive anti-ds DNA response was mostly IgM with little IgG subclass switching, indicating no affinity maturation or memory induction, characteristics 


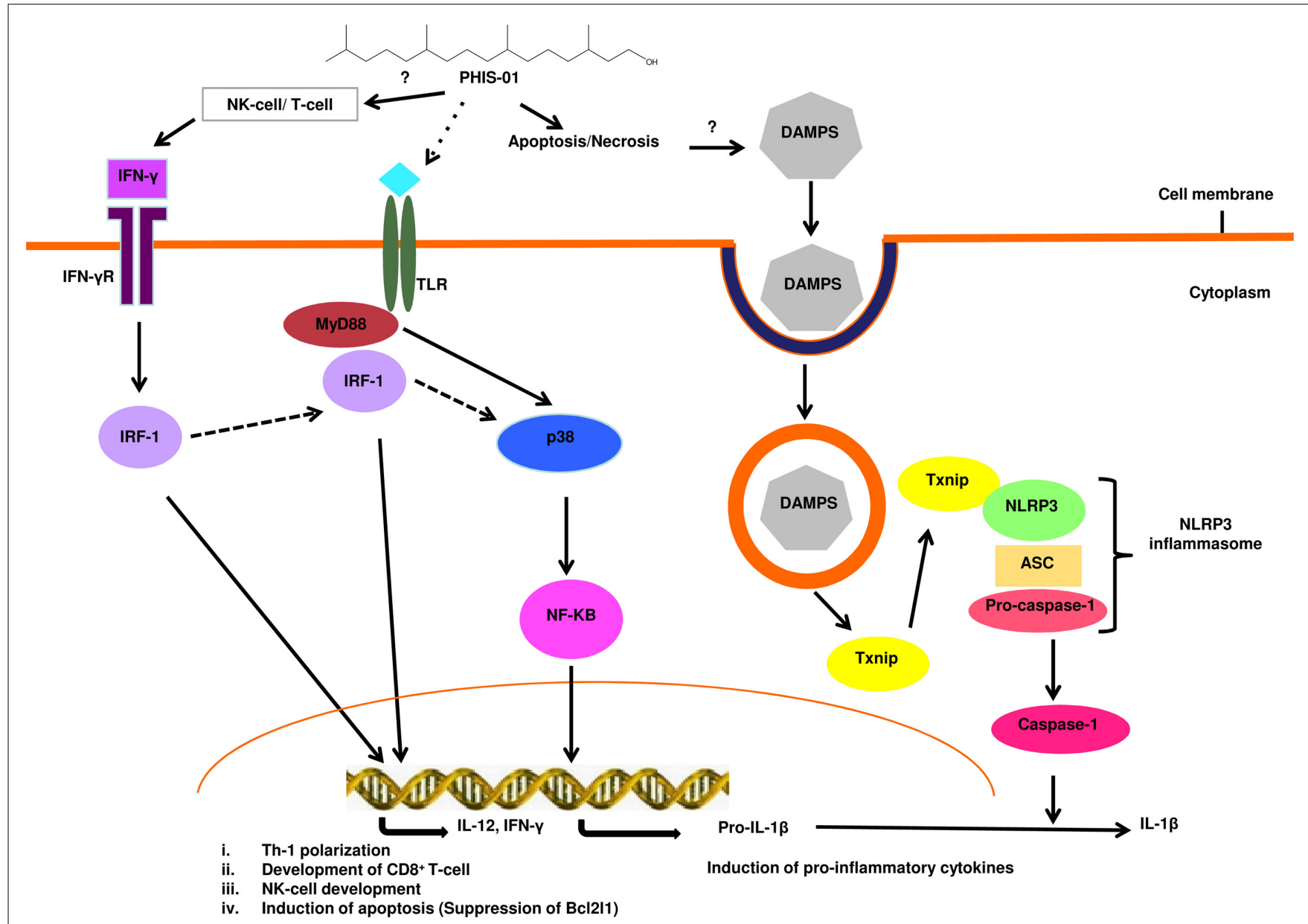

FIGURE 3 | Possible signaling pathway activated by the phytol-based immunostimulant PHIS-01. PHIS-01 induces IFN- $\gamma$, leading to the activation of the transcription factor IRF-1 that transcribes genes resulting in Th1 polarization, cytotoxic T-lymphocyte

development, induction of apoptosis and also possibly the activation of
NK cells. PHIS-01 induced apoptosis/necrosis can generate DAMPS leading to the activation of the Nlrp3 inflammasome and production of inflammatory cytokines. MyD88 and p38 mediated activation of NF-KB is probably induced by PHIS-01 indirectly via the activation of Toll-like or Nod-like receptors. of T helper activity on antigen-specific B cells (Ghosh and Aachoui, 2011).

\section{DISCUSSION}

The overall objective of this review was to provide comparative pictures of both commercial and phytol-based synthetic adjuvants. Our findings support the contention that phytol-derived adjuvants are safe and efficacious immunostimulants. This conclusion can be drawn on the basis of their ability to promote effective humoral response, stimulate $\mathrm{T}$ cell proliferation without exhibiting any adverse autoimmune anti-DNA response in resistant and susceptible mice strains. Future studies with selective chemical modifications of simple diterpenoids like phytol, followed by assessment of adjuvanticity, may unlock key structural features necessary for effectiveness of such immunostimulants.

The rewarding aspect of adjuvant research is that it not only paves the way to improving current technology for vaccine development, but also addresses basic questions relating to immunological memory. One current thought is that some fragments of immunogenic substances are retained by APCs in order to sustain immune memory in B and T cells; these APCs possibly upregulate BCL2, BCL-XL, and similar anti-apoptotic transcription factors. It is not clear how adjuvants augment immunogenicity and efficacy of vaccines. It is possible that adjuvants directly work on anti-apoptotic molecules or sustain a chronic inflammatory cytokine milieu favorable for retention of memory cells. It may also be possible to structurally modify and design adjuvants that selectively favor the induction of specific classes of antibody molecules, such as non-complement fixing IgG4. Using phytolderived diterpenoids we hope to address some of these questions in future.

\section{ACKNOWLEDGMENTS}

This work was supported by funds from grants provided by Fraternal Order of Eagles, Indiana Academy of Science (SAC 131 to Swapan K. Ghosh and PAC 045 to Roshni Roy Chowdhury), the Indiana State Research Committee (UNR 319), and graduate student funding from Indiana State University. 


\section{REFERENCES}

Aachoui, Y. (2011). Novel PhytolDerived Immunostimulants (PHIS01) for Enhancement of Vaccine Efficacy: A Comparative Study. Dissertation, Indiana State University, Terre Haute.

Aachoui, Y., Schulte, M. L., Fitch, R. W., and Ghosh, S. K. (2011a). Synthetic adjuvants for vaccine formulations: evaluation of new phytol derivatives in induction and persistence of specific immune response. Cell. Immunol. 271, 308-318.

Aachoui, Y., Chowdhury, R. R., Fitch, R. W., and Ghosh, S. K. (2011b). Ghosh molecular signatures of phytol-derived immunostimulants in the context of chemokinecytokine microenvironment and enhanced immune response. Cell. Immunol. 271, 227-238.

Allan, S. (2008). Vaccines: explaining alum: immunologists' dirty little secret. Nat. Rev. Immunol. 8, 320.

Asa, P. B., Cao, Y., and Garry, R. F. (2000). Antibodies to squalene in Gulf War syndrome. Exp. Mol. Pathol. 68, 55-64.

Azuma, I. (1992). Synthetic immunoadjuvants: application to non-specific host stimulation and potentiation of vaccine immunogenicity. Vaccine 10 , 1000-1006.

Bakkali, F., Averbeck, S., Averbeck, D., and Idaomar, M. (2008). Idaomar biological effects of essential oils a review. Food Chem. Toxicol. 46, 446-475.

Banzhoff, A., Gasparini, R., LaghiPasini, F., Staniscia, T., Durando, P., Montomoli, E., Capecchi, P. L., di Giovanni, P., Sticchi, L., Gentile, C., Hilbert, A., Brauer, V., Tilman, S., and Podda, A. (2009). MF59adjuvanted $\mathrm{H} 5 \mathrm{~N} 1$ vaccine induces immunologic memory and heterotypic antibody responses in nonelderly and elderly adults. PLoS ONE 4, e4384. doi:10.1371/journal.pone.0004384

Banzhoff, A., Pellegrini, M., Del Giudice, G., Fragapane, E., Groth, N., and Podda, A. (2008). MF59adjuvanted vaccines for seasonal and pandemic influenza prophylaxis. Influenza Other Respi. Viruses 2, 243-249.

Baudner, B. C., Ronconi, V., Casini, D., Tortoli, M., Kazzaz, J., Singh, M., Hawkins, L. D., Wack, A., and O'Hagan, D. T. (2009). MF59 emulsion is an effective delivery system for a synthetic TLR4 agonist (E6020). Pharm. Res. 26, 1477-1485.

Baxter, J. H. (1968). Absorption of chlorophyll phytol in normal man and in patients with Refsum's disease. J. Lipid Res. 9, 636-641.

Benísek, Z., Süli, J., Eliás, D., Lenhardt, L., Ondrejková, A., Ondrejka, R., Svrcek, S., and Bajová, V. (2004). Experimental squalene adjuvant II. Harmlessness and local reactogenicity. Vaccine 22, 3470-3474.

Bifulco, M. (2005). Role of the isoprenoid pathway in ras transforming activity, cytoskeleton organization, cell proliferation and apoptosis. Life Sci. 77, 1740-1749.

Billiau, A., and Matthys, P. (2001). Modes of action of Freund's adjuvants in experimental models of autoimmune diseases. J. Leukoc. Biol. 70, 849-860.

Blagowechensky, N. (1938). Durée du séjour de l'antigène dans l'organisme et immunité. Rev. Immunol. 4, 161.

Brewer, J. M., Conacher, M., Hunter, C. A., Mohrs, M., Brombacher, F., and Alexander, J. (1999). Aluminium hydroxide adjuvant initiates strong antigen-specific Th2 responses in the absence of IL-4- or IL-13mediated signaling. J. Immunol. 163, 6448-6454.

Butler, N. R., Voyce, M. A., Burland, W. L., and Hilton, M. L. (1969). Advantages of aluminium hydroxide adsorbed combined diphtheria, tetanus, and pertussis vaccines for the immunization of infants. $B r$. Med. J. 1, 663-666.

Casadevall, A., and Pirofski, L. A. (1998). Use of licensed vaccines for active immunization of the immunocompromised host. Clin. Microbiol. Rev. 11, 1-26.

Cox, J. C., Sjölander, A., and Barr, I. G. (1998). ISCOMs and other saponin based adjuvants. Adv. Drug Deliv. Rev. 32, 247-271.

Cribbs, D. H., Ghochikyan, A., Vasilevko, V., Tran, M., Petrushina, I., Sadzikava, N., Babikyan, D., Kesslak, P., Kieber-Emmons, T., Cotman, C. W., and Agadjanyan, M. G. (2003). Adjuvant dependent modulation of Th1 and Th2 responses to immunization with $\beta$ amyloid. Int. Immunol. 15, 505-514.

Didierlaurent, A. M., Morel, S., Lockman, L., Giannini, S. L., Bisteau, M., Carlsen, H., Kielland, A., Vosters, O., Vanderheyde, N., Schiavetti, F., Larocque, D., Van Mechelen, M., and Garçon, N. (2009). AS04, an aluminum salt- and TLR4 agonistbased adjuvant system, induces a transient localized innate immune response leading to enhanced adaptive immunity. J. Immunol. 183, 6186-6197.
Dvorak, A. M., and Dvorak, H. F. (1974). Structure of Freund's complete and incomplete adjuvants. Relation of adjuvanticity to structure. Immunology 27, 99-114.

Elson, C. E., and Yu, S. G. (1994) The chemoprevention of cancer by mevalonate-derived constituents of fruits and vegetables. J. Nutr. 124, 607-614.

Estrada, A., Li, B., and Laarveld, B. (1998). Adjuvant action of Chenopodium quinoa saponins on the induction of antibody responses to intragastric and intranasal administered antigens in mice. Comp. Immunol. Microbiol. Infect. Dis. 21, 225-236.

Geiera, D. A., King, P. G., and Geierc, M. R. (2009). Mitochondrial dysfunction, impaired oxidative-reduction activity, degeneration, and death in human neuronal and fetal cells induced by low-level exposure to thimerosal and other metal compounds. Toxicol. Environ. Chem. 91, 735-749.

Ghosh, S. K. (2012). Phytol derived immunoadjuvants and their use in vaccine formulations. United States Patent 8088395

Ghosh, S. K., and Aachoui, Y. (2011). Immune enhancement by novel vaccine adjuvants in autoimmuneprone NZB/W F1 mice: relative efficacy and safety. BMC Immunol. 12, 61. doi:10.1186/1471-2172-12-61

Glomset, J. A., Gelb, M. H., and Farnsworth, C. C. (1990). Prenyl proteins in eukaryotic cells: a new type of membrane anchor. Trends Biochem. Sci. 15, 139-142.

Granoff, D. M., McHugh, Y. E., Raff, H. V., Mokatrin, A. S., and Van Nest, G. A. (1997). MF59 adjuvant enhances antibody responses of infant baboons immunized with Haemophilus influenzae type $\mathrm{b}$ and Neisseria meningitidis group C oligosaccharide-CRM197 conjugate vaccine. Infect. Immun. 65, 1710-1715.

Gupta, R. K., Relyveld, E. H., Lindblad, E. B., Bizzini, B., Ben-Efraim, S., and Gupta, C. K. (1993). Adjuvants - a balance between toxicity and adjuvanticity. Vaccine 11, 293-306.

Harrewijn, P., van Oosten, A. M., and Piron, P. G. M. (2001). Natural terpenoids as messengers. A multidisciplinary study of their production, biological functions and practical applications. Ann. Bot. 90, 299-300.

Heinemann, L., Woodfield, L., Amer, M., and Hibma, M. (2008). Effective induction of type 1 helper IgG2a and cytotoxic T-cell responses in mice following immunization with human papillomavirus type 16 E2 in MF59. Viral Immunol. 21, 225-233.

Holm, B. C., Lorentzen, J. C., and Bucht, A. (2004). Adjuvant oil induces waves of arthritogenic lymph node cells prior to arthritis onset. Clin. Exp. Immunol. 137, 59-64.

Holmdahl, R., Lorentzen, J. C., Lu, S., Olofsson, P., Wester, L., Holmberg, J., and Pettersson, U. (2001). Arthritis induced in rats with nonimmunogenic adjuvants as models for rheumatoid arthritis. Immunol. Rev. 184, 184-202.

Isman, M. B. (2006). Botanical insecticides, deterrents, and repellents in modern agriculture and an increasingly regulated world. Annu. Rev. Entomol. 51, 45-66.

Janeway, C. (1989). Approaching the asymptote? Evolution and revolution in immunology. Cold Spring Harb. Symp. Quant. Biol. 54, 1-13.

Kagoura, M., Matsui, C., and Morohashi, M. (1999). Phytol is a novel tumor promoter on ICR mouse skin. Jpn. J. Cancer Res. 4, 377-384.

Kalvodova, L. (2010). Squalene-based oil-in-water emulsion adjuvants perturb metabolism of neutral lipids and enhance lipid droplet formation. Biochem. Biophys. Res. Commun. 393, 350-355.

Kazanietz, M. (2005). Targeting protein kinase C and "non-kinase" phorbol ester receptors: emerging concepts and therapeutic implications. Biochim. Biophys. Acta 1754, 296-304.

Kensil, C. R., and Kammer, R. (1998). QS-21: a water-soluble triterpene glycoside adjuvant. Expert Opin. Investig. Drugs 7, 1475-1482.

Kensil, C. R., Liu, G., Anderson, C., and Storey, J. (2006). Effects of QS21 on innate and adaptive immune responses. Vaccin. Adjuvants 221-234.

Kono, H., and Rock, K. L. (2008). How dying cells alert the immune system to danger. Nat. Rev. Immunol. 8, 279-289.

Krishnan, L., Dicaire, C. J., Patel, G. B., and Sprott, G. D. (2000). Archaeosome vaccine adjuvants induce strong humoral, cell-mediated, and memory responses: comparison to conventional liposomes and alum. Infect. Immun. 68, 54-63.

Kuroda, Y., Nacionales, D. C., Akaogi, J., Reeves, W. H., and Satoh, M. (2004). Autoimmunity induced by adjuvant hydrocarbon oil components of vaccine. Biomed. Pharmacother. 58, 325-337.

Lederer, E. (1980). Synthetic immunostimulants derived from the 
bacterial cell wall. J. Med. Chem. 8, 819-825.

Lee, I. F., van den Elzen, P., Tan, R., and Priatel, J. J. (2011). NKT cells are required for complete Freund's adjuvant-mediated protection from autoimmune diabetes. J. Immunol. 187, 2898-2904.

Lévesque, S., Martinez, G., and Fairbrother, J. M. (2007). Improvement of adjuvant systems to obtain a costeffective production of high levels of specific IgY. Poult. Sci. 86, 630-635.

$\mathrm{Li}, \mathrm{H}$. (2009). Impact of ImmunoAdjuvants on Anti-Lymphoma Vaccine Efficacy. Ph.D. thesis, Indiana State University, Terre Haute.

Li, H., Willingham, S. B., Ting, J. P., and Re, F. (2008). Cutting edge: inflammasome activation by alum and alum's adjuvant effect are mediated by NLRP3. J. Immunol. 181, $17-21$

Lim, S.-K. (2003). Freund adjuvant induces TLR2 but not TLR4 expression in the liver of mice. Int. Immunopharmacol. 3, 115-118.

Lim, S. Y., and Ghosh, S. K. (2003). Autoreactive responses to an environmental factor: 1 phthalate induces antibodies exhibiting antiDNA specificity. Immunology 110, 482-492.

Lim, S. Y., and Ghosh, S. K. (2004). Autoreactive responses to environmental factors: 3. Mouse strainspecific differences in induction and regulation of anti-DNA antibody responses due to phthalate-isomers. J. Autoimmun. 25, 33-45.

Lim, S. Y., Meyer, M., Kjonaas, R. A., and Ghosh, S. K. (2006a). Phytolbased novel adjuvants in vaccine formulation 1 assessment of safety and efficacy during stimulation of humoral and cell-mediated immune responses. J. Immune Based Ther. Vaccines 4, 5.

Lim, S. Y., Bauermeister, A., Kjonaas, R. A., and Ghosh, S. K. (2006b). Phytolbased novel adjuvants in vaccine formulation: 2 assessment of efficacy in the induction of protective immune responses to lethal bacterial infections in mice. J. Immune Based Ther. Vaccines 4, 5.

Lindblad, E. (1995). Aluminium adjuvants. The Theory and Practical Application of Adjuvants. Chichester: John Wiley \& Sons Ltd., 21-35.

Maa, Y. F., Zhao, L., Payne, L. G., and Chen, D. (2002). Stabilization of alum-adjuvanted vaccine dry powder formulations: mechanism and application. J. Pharm. Sci. 92, 319-332.

Maimone, T. J., and Baran, P. S. (2007). Modern synthetic efforts toward biologically active terpenes. Nat. Chem. Biol. 3, 396-407.

Marchkesbery, W. R., Ehmann, W. D., Hossain, T. I., Alauddin, M., and Goodin, D. T. (1981). Instrumental neutron activation analysis of brain aluminum in Alzheimer disease and aging. Ann. Neurol. 10, 511-516.

Marciani, D. J., Pathak, A. K., Reynolds, R. C., Seitz, L., and May, R. D. (2001). Altered immunomodulating and toxicological properties of degraded Quillaja saponaria Molina saponins. Int. Immunopharmacol. 1, 813-818.

Marciani, D. J., Press, J. B., Reynolds, R. C., Pathak, A. K., Pathak, V., Gundy, L. E., Farmer, J. T., Koratich, M. S., and May, R. D. (2000). Development of semisynthetic triterpenoid saponin derivatives with immune stimulating activity. Vaccine 18, 3141-3151.

Marrack, P., McKee, A. S., and Munks, M. W. (2009). Towards an understanding of the adjuvant action of aluminium. Nat. Rev. Immunol. 9, 287-293.

Mastelic, B., Ahmed, S., Egan, W. M., Del Giudice, G., Golding, H., Gust, I., Neels, P., Reed, S. G., Sheets, R. L., Siegrist, C. A., and Lambert, P. H. (2010). Mode of action of adjuvants: implications for vaccine safety and design. Biologicals 38 , 594-601.

Matthys, P., Vermeire, K., Mitera, T., Heremans, H., Huang, S., Schols, D., De Wolf-Peeters, C., and Billiau, A. (1999). Enhanced autoimmune arthritis in IFN-gamma receptordeficient mice is conditioned by mycobacteria in Freund's adjuvant and by increased expansion of Mac$1+$ myeloid cells. J. Immunol. 163, 3503-3510.

Mbow, M. L., De Gregorio, E., and Ulmer, J. B. (2011). Alum's adjuvant action: grease is the word. Nat. Med. $17,415-416$.

McInerney, M. F., Pek, S. B., and Thomas, D. W. (1991). Prevention of insulitis and diabetes onset by treatment with complete Freund's adjuvant in NOD mice. Diabetes 40, 715-725.

McKee, A. S., MacLeod, M. K., Kappler, J. W., and Marrack, P. (2010). Immune mechanisms of protection: can adjuvants rise to the challenge? BMC Biol. 8, 37. doi:10.1186/1741-7007-8-37

Medzhitov, R., and Pasare, C. (2003). Toll pathway-dependent blockade of $\mathrm{CD} 4+\mathrm{CD} 25+\mathrm{T}$ cell-mediated suppression by dendritic cells. Science 299, 1033-1036.
Mo, H., and Elson, C. E. (2004). Studies of the isoprenoid-mediated inhibition of mevalonate synthesis applied to cancer chemotherapy and chemoprevention. Exp. Biol. Med. (Maywood) 229, 567-585.

Montagnani, S., Tuccori, M., Lombardo, G., Testi, A., Mantarro, S., Ruggiero, E., and Blandizzi, C. (2011). Autoimmune hemolytic anemia following MF59-adjuvanted influenza vaccine administration: a report of two cases. Ann. Pharmacother. 45, e8.

Mosca, F., Tritto, E., Muzzi, A. Monaci, E., Bagnoli, F., Iavarone, C., O'Hagan, D., Rappuoli, R., and De Gregorio, E. (2008). Molecular and cellular signatures of human vaccine adjuvants. Proc. Natl. Acad. Sci. U.S.A. 105, 10501-10506.

Norton, E. B., Clements, J. D., Voss, T. G., and Cárdenas-Freytag, L. (2010). Prophylactic Administration of bacterially derived immunomodulators improves the outcome of influenza virus infection in a murine model. $J$. Virol. 84, 2983-2995.

Oda, K., Matsuda, H., Murakami, T., Katayama, S., Ohgitani, T., and Yoshikawa, M. (2000). Adjuvant and haemolytic activities of 47 saponins derived from medicinal and food plants. Biol. Chem. 381, 67-74.

Oscherwitz, J., Hankenson, F. C., Yu, F., and Cease, K. B. (2006). Lowdose intraperitoneal Freund's adjuvant: toxicity and immunogenicity in mice using an immunogen targeting amyloid- $\beta$ peptide. Vaccine 24 , 3018-3025.

Ott, G., Singh, M., Kazzaz, J., Briones, M., Soenawan, E., Ugozzoli, M., and O'Hagan, D. T. (2002). A cationic sub-micron emulsion (MF59/DOTAP) is an effective delivery system for DNA vaccines. $J$. Control. Release 79, 1-5.

Pasare, C., and Medzhitov, R. (2004). Toll-dependent control mechanisms of CD4 T cell activation. Immunity 21, 733-741.

Peng, S. L., Moslehi, J., and Craft, J. (1997). Roles of interferon-gamma and interleukin-4 in murine lupus. J. Clin. Invest. 99, 1936-1946.

Petrovsky, N., and Aguilar, J. C. (2004). Vaccine adjuvants: current state and future trends. Immunol. Cell Biol. 82, 488-496.

Pickett, J. A., and Gibson, R. W. (1983). Wild potato repels aphids by release of aphid alarm pheromone. Nature 302, 608-609.

Raghavendra, V., Tanga, F. Y., and DeLeo, J. A. (2004). Complete Freunds adjuvant-induced peripheral inflammation evokes glial activation and proinflammatory cytokine expression in the CNS. Eur. J. Neurosci. 20, 467-473.

Rajput, Z. I., Hu, S. H., Xiao, C. W., and Arijo, A. G. (2007). Adjuvant effects of saponins on animal immune responses. J. Zhejiang Univ. Sci. B 8, 153-161.

Reddy, L. H., and Couvreur, P. (2009). Squalene: a natural triterpene for use in disease management and therapy. Adv. Drug Deliv. Rev. 61, 1412-1426.

Reeves, W. H., Lee, P. Y., Weinstein, J. S., Satoh, M., and Lu, L. (2009). Induction of autoimmunity by pristane and other naturally-occurring hydrocarbons. Trends Immunol. 30, 455-464.

Rhodes, J., Chen, H., Hall, S. R., Beesley, J. E., Jenkins, D. C., Collins, P., and Zheng, B. (1995). Therapeutic potentiation of the immune system by costimulatory Schiff-baseforming drugs. Nature 377, 71-75.

Rilling, H. C., Breunger, E., Epstein, W. W., and Crain, P. F. (1990). Prenylated proteins: the structure of the isoprenoid group. Science 247, 318-320.

Salkowski, C. A., Detore, G. R., and Vogel, S. N. (1997). Lipopolysaccharide and monophosphoryl lipid A differentially regulate interleukin-12, gamma interferon, and interleukin-10 mRNA production in murine macrophages. Infect. Immun. 65, 3239.

Satoh, M., Kuroda, Y., Yoshida, H., Behney, K. M., Mizutani, A., Akaogi, J., Nacionales, D. C., Lorenson, T. D., Rosenbauer, R. J., and Reeves, W. H. (2003). Induction of lupus autoantibodies by adjuvants. J. Autoimmun 21, 1-9.

Seubert, A., Calabro, S., Santini, L., Galli, B., Genovese, A., Valentini, S., Aprea, S., Colaprico, A., D’Oro, U., Giuliani, M. M., Pallaoro, M., Pizza, M., O'Hagan, D. T., Wack, A., Rappuoli, R., and De Gregorio, E. (2011). Adjuvanticity of the oil-inwater emulsion MF59 is independent of Nlrp3 inflammasome but requires the adaptor protein MyD88. Proc. Natl. Acad. Sci. U.S.A.108, 11169-11174.

Seubert, A., Monaci, E., Pizza, M., O'Hagan, D. T., and Wack, A. (2008) The adjuvants aluminum hydroxide and MF59 induce monocyte and granulocyte chemoattractants and enhance monocyte differentiation toward dendritic cells. J. Immunol. 180, 5402-5412.

Shieh, H. L., Hansen, H., Zhu, J., and Riedel, H. (1995). Differential protein kinase $\mathrm{C}$ ligand regulation detected in vivo by a phenotypic 
yeast assay. Mol. Carcinog. 12, Sparg, S. G., Light, M. E., and van $166-176$.

Skene, C. D., and Sutton, P. (2006). Saponin-adjuvanted particulate vaccines for clinical use. Methods 40, 53-59.

Smith, K. M., Brewer, J. M., Rush, C. M., Riley, J., and Garside, P. (2004). In vivo generated Th1 cells can migrate to B cell follicles to support B cell responses. J. Immunol. 173, 1640-1646.

Soltysik, S., Wu, J. Y., Recchia, J., Wheeler, D. A., Newman, M. J., Coughlin, R. T., and Kensil, C. R. (1995). Structure/function studies of QS-21 adjuvant: assessment of triterpene aldehyde and glucuronic acid roles in adjuvant function. Vaccine 13, 1403-1410.

Song, X., and Hu, S. (2009). Adjuvant activities of saponins from traditional Chinese medicinal herbs. Vaccine 27, 4883-4890.

Spanedda, M. V., Heurtault, B., Weidner, S., Baehr, C., Boeglin, E., Beyrath, J., Milosevic, S., Bourel-Bonnet, L., Fournel, S., and Frisch, B. (2010). Novel powerful water-soluble lipid immunoadjuvants inducing mouse dendritic cell maturation and $B$ cell proliferation using TLR2 pathway. Bioorg. Med. Chem. Lett. 20, 1869-1872.

Staden, J. (2004). Biological activities and distribution of plant saponins. J. Ethnopharmacol. 94, 219-243.

Staats, H. F., and Ennis, F. A. Jr. (1999). IL-1 Is an effective adjuvant for mucosal and systemic immune responses when coadministered with protein immunogens. J. Immunol. 162, 6141-6147.

Stills, H. F. Jr. (2005). Adjuvants and antibody production: dispelling the myths associated with Freund's complete and other adjuvants. ILAR J. 46, 280-293.

Straw, B. E., MacLachlan, N. J., Corbett, W. T., Carter, P. B., and Schey, H. M. (1985). Comparison of tissue reactions produced by Haemophilus pleuropneumoniae vaccines made with six different adjuvants in swine. Can. J. Comp. Med. 49, 149-151.

Strominger, J. L. (2007). Bacterial cell walls, innate immunity and immunoadjuvants. Nat. Immunol. 8, 1269-1271.

Sun, H. X., Xie, Y., and Ye, Y. P. (2009). Advances in saponinbased adjuvants. Vaccine 27, 1787-1796.

Thompson, B. S., Chilton, P. M., Ward, J. R., Evans, J. T., and Mitchell, T. C. (2005). The low-toxicity versions of
LPS, MPL adjuvant and RC529, are efficient adjuvants for CD4 T cells. $J$. Leukoc. Biol. 78, 1273-1280.

induce autoimmune arthritis in mice. Immunology 4, 607-614.

Zwenger, S., and Basu, C. (2008). Plant terpenoids: applications and future potentials. Biotechnol. Mol. Biol. Rev. 3, 1-7.

Terpenoids as potential chemopreventive and therapeutic agents in liver cancer. World J. Hepatol. 3, 228-249.

Traquina, P., Morandi, M., Contorni, M., and Van Nest, G. (1996). MF59 adjuvant enhances the antibody response to recombinant hepatitis B surface antigen vaccine in primates. J. Infect. Dis. 174, 1168-1175.

Wilner, B. I., Evers, M. A., Troutman, H. D., Trader, F. W., and Mclean, I. W. (1963). Vaccine potentiation by emulsification with pure hydrocarbon compounds. J. Immunol. 91, 210-229.

Yip, H. C., Karulin, A. Y., TaryLehmann, M., Hesse, M. D. Radeke, H., Heeger, P. S., Trezza, R. P., Heinzel, F. P., Forsthuber, T., and Lehmann, P. V. (1999). Adjuvant-guided type-1 and type-2 immunity: infectious/noninfectious dichotomy defines the class of response. J. Immunol. 62, 3942-3949.

Yoshino, S., Sasatomi, E., and Ohsawa, M. (2000). Bacterial lipopolysaccharide acts as an adjuvant to
Conflict of Interest Statement: The authors declare that the research was conducted in the absence of any commercial or financial relationships that could be construed as a potential conflict of interest.

Received: 31 January 2012; paperpending published: 21 February 2012; accepted: 28 February 2012; published online: 22 March 2012.

Citation: Chowdhury RR and Ghosh SK (2012) Phytol-derived novel isoprenoid immunostimulants. Front. Immun. 3:49. doi: 10.3389/fimmu.2012.00049

This article was submitted to Frontiers in Immunotherapies and Vaccines, a specialty of Frontiers in Immunology. Copyright (c) 2012 Chowdhury and Ghosh. This is an open-access article distributed under the terms of the Creative Commons Attribution Non Commercial License, which permits noncommercial use, distribution, and reproduction in other forums, provided the original authors and source are credited. 